\title{
Patient mortality of active pulmonary tuberculosis requiring mechanical ventilation
}

\author{
P.L. Lee*, J.S. Jerng*, Y.L. Chang*, C.F. Chen ${ }^{\Uparrow}$, P.R. Hsueh ${ }^{+}$, C.J. Yu*, P.C. Yang*, K.T. Luh ${ }^{+}$
}

Patient mortality of active pulmonary tuberculosis requiring mechanical ventilation. P.L. Lee, J.S. Jerng, Y.L. Chang, C.F. Chen, P.R. Hsueh, C.J. Yu, P.C. Yang, K.T. Luh. (C) ERS Journals Ltd 2003.

ABSTRACT: Mortality remains high among patients with pulmonary tuberculosis requiring mechanical ventilation (TBMV). This study was carried out to establish the mortality rates of TBMV and to identify factors that contribute to in-hospital mortality.

From January 1996-April 2001, there were 825 patients with active pulmonary tuberculosis at the National Taiwan University Hospital, Taipei, Taiwan. Of these, 41 suffered acute respiratory failure and required mechanical ventilation in the intensive care unit (ICU). Of these 41 patients, 38 were followed up for 180 days. In-hospital deaths were documented in the medical records and all possible parameters contributing to mortality were collected.

Of the 41 patients, 27 died in the hospital and 14 were discharged alive (in-hospital mortality rate $65.9 \%$ ), with (mean \pm SD) $40.7 \pm 35.4$ admission days before death. Of the 27 that died, 25 died during ICU admission and two died after being transferred to the ward. The mortality rate for the 180 -day monitoring period was $79 \%$. Factors contributing to in-hospital mortality included consolidations on chest radiographs and multiple organ failure.

The mortality rate in the patients with pulmonary tuberculosis requiring mechanical ventilation is very high, with two factors affecting in-hospital mortality. These factors were multiple organ failure and consolidation on chest radiographs.

Eur Respir J 2003; 22: 141-147.
*Dept of Internal Medicine, ${ }^{\text {\#Dept of Patho- }}$ logy and ${ }^{+}$Dept of Laboratory Medicine, National Taiwan University Hospital, Taipei, and Dept of Health Administration, Hung Kuang Institute of Technology, Taiwan.

Correspondence: C.J. Yu

Dept of Internal Medicine

National Taiwan University Hospital

7 Chung-Shan South Road

Taipei 100

Taiwan

Fax: 886223582867

E-mail: jeffery@ha.mc.ntu.edu.tw

Keywords: Mechanical ventilation pulmonary tuberculosis respiratory failure

Received: May 92002

Accepted after revision: January 312003
In 2000, an estimated 8 million people were infected and 2 million people died of tuberculosis (TB) worldwide [1-3]. Despite advances in chemotherapy, cases of pulmonary TB and TB-related critical illness increased dramatically over the last few decades. In-hospital mortality rate remains high at $\sim 60 \%$, for patients with active pulmonary TB and respiratory failure [4-10]. This is twice as high as the mortality rate of patients with pneumonia requiring mechanical ventilation $[4$, $11,12]$.

Delays in the diagnosis and treatment of pulmonary TB have been recognised as the main causes of death $[2,4,8$, 13-16]. Several studies have sought to reduce these delays by identifying factors that lead to a high index of suspicion $[4,8$, $13-15,17-20]$. The reasons for delays in diagnosis include the failure to perform appropriate diagnostic investigation and the misinterpretation of radiological or clinical manifestations as malignancy, pneumonia or other conditions [4, 8, 13-15, 17-22].

Reports of active pulmonary TB with acute respiratory failure are not scarce, but few focus on mechanically ventilated patients. Such patients have a higher mortality rate than patients who do not need mechanical ventilation [4-6, 8, 10]. However, $>50 \%$ of the patients included in these previous studies were positive for human immunodeficiency virus (HIV) infection. Hence, conclusions derived from these studies may not apply to HIV-negative patients.

Therefore, the authors conducted a retrospective study with three objectives in relation to active pulmonary TB patients requiring support by mechanical ventilation (TBMV). These objectives were: 1) to identify specific patterns in pulmonary TB that will enable physicians to have a high index of suspicion; 2) to establish mortality rates of TBMV; and 3) to identify factors contributing to in-hospital mortality.

\section{Material and methods}

\section{Subjects}

Medical records from January 1996-April 2001 were reviewed for all of the patients diagnosed with pulmonary TB at the National Taiwan University Hospital, Taipei, Taiwan, a tertiary-care transfer centre. Sputum culture showed that 825 patients were positive for Mycobacterium tuberculosis. Of the 825 patients, 41 had acute respiratory failure and required mechanical ventilation in the intensive care unit (ICU). These 41 patients constituted the study group.

\section{Methods}

Clinical data were obtained from medical records and categorised as follows: demographic characteristics, disease presentations and investigation, and outcomes. These data included sex, age, comorbidities, coexisting pulmonary diseases, chronic health status, symptoms, time from onset of symptomatology to treatment, characteristics of the TB (pneumonic or 
miliary TB), the presence of extrapulmonary TB, chest radiographic patterns and ICU admission parameters (Acute Physiology and Chronic Health Evaluation (APACHE) II scores, numbers of organ failures).

Laboratory tests investigated included white cell count, HIV status, sputum smears and cultures, drug sensitivity of $M$. tuberculosis isolates, arterial blood gas and blood culture. Other data obtained also included parameters of lung mechanics (static airway resistance, static compliance), tentative diagnosis, treatments (anti-TB therapy, time to anti-TB therapy from admission, steroids for adult respiratory distress syndrome (ARDS)), investigations (bronchoscopy, biopsy) and outcomes (duration of follow-up, hospital and ICU length of stay, hospital and ICU mortality, duration of mechanical ventilation and cause of death).

Mortality during the follow-up period was determined using a combined review of the medical records and telephone follow-up. Results were further confirmed using the death certificate registry of the Department of Health, Taiwan. Other follow-up information was obtained by telephone interview and a review of medical records.

Miliary TB was defined as the presence of micronodules on chest radiographs or high-resolution computed tomography. Conversely, pneumonic TB was defined as nonmiliary parenchymal pulmonary TB. Disseminated TB was defined as active TB without a miliary pattern, but with the involvement of at least two extrapulmonary sites. Chest radiographs obtained during the admission procedure were reviewed by one pulmonologist and were classified as nodular, consolidation, interstitial or cavitary. The extent of lung involvement was classified as $\leqslant 2$ or $\geqslant 3$ quadrants.

ARDS was diagnosed based on the consensus of the American-European conference [23]. Organ failure was diagnosed based on the criteria created by Knaus [24, 25], while multiple organ failure (MOF) was defined as the failure of more than one organ. Resistance to first-line agents was determined using critical concentrations in a liquid media and resistance to second-line drugs was similarly determined on Lowenstein-Jenssen slants. Multidrug resistance (MDR) was defined as resistance to at least isoniazid and rifampin.

Tentative diagnosis was defined as the primary diagnosis made when patients arrived at the hospital. Anti-TB therapy was defined as the administration of at least two of the first-line (isoniazid, rifampin, pyrazinamide, ethambutol and streptomycin) and/or second-line anti-TB drugs. A delay in the treatment of TB was identified as anti-TB therapy not initiated within $24 \mathrm{~h}$ following hospital admission. The duration of the delay was counted in days from the day of admission to the start of the anti-TB therapy. Specimens from patients who had undergone lung biopsy were reviewed by a pathologist and classified as the presence of acid-fast bacilli or giant cells, caseous necrosis, granuloma, chronic inflammation or fibrosis.

\section{Statistical analysis}

The data were compared between the group of patients who died and the group who survived during hospitalisation. Continuous variables were compared using an unpaired t-test. Chi-squared or Fisher's exact tests were used to compare categorical variables. All of the values were expressed as mean \pm SD for continuous variables and percentage of the group for categorical variables.

Variables in the univariate analysis with a $\mathrm{p}<0.05$ were considered as having statistically significant association with death. Kaplan-Meier survival curves were used to analyse the survival of patients. Factors influencing survival were compared with the log-rank test. Those factors significantly associated with survival were further analysed with the Coxproportional hazard model to adjust for potential confounding effects between each other factor. Hazard ratio (HR) with $95 \%$ confidence interval (CI) was used to report the result.

\section{Results}

A total of 41 patients were included in the study. Of the 41 patients, 38 were followed for 180 days. Three were followedup for shorter periods of 53, 65 and 60 days, due to loss of contact thereafter.

\section{Demographic characteristics}

Table 1 is the list of the demographic characteristics of the 41 patients. The mean age of all patients was $62 \mathrm{yrs}$; the difference between the survivors and those that died was not statistically significant. Males outnumbered females, especially among the survivors. None of the 41 patients were positive for HIV infection. Less than one-half of the patients $(n=20)$ had underlying illness that would increase the risk of pulmonary TB. Diabetes was the most common comorbidity. Eleven $(27 \%)$ of the patients had other underlying pulmonary diseases: five had pneumoconiosis, four had obstructive lung disease and two had interstitial lung disease. However, these 11 patients did not need oxygen therapy before this episode.

\section{Disease presentations}

Table 1 shows the characteristics of disease presentations. Dyspnoea was the most common symptom, instead of cough and fever. The mean duration from symptoms to admission was short, 9.0 11.6 days (range 1-60 days). There were twice as many pneumonic TB cases as miliary TB. Ten patients $(24 \%)$ had TB involving extrapulmonary sites: the pleura in five, brain abscess in two, lymph node, blood and bone marrow in one, bone marrow in one and muscle in one. Only one had disseminated TB. Most patients were in critical condition with mean APACHE II scores above 16 and with the presence of more than two failed organs.

\section{Radiological features and laboratory parameters}

Tables 2 and 3 show the radiological features and laboratory parameters, respectively. The most common radiographic finding was consolidation $\geqslant 3$ quadrants. Cavitation was present in only one-third of the patients $(n=15)$. In 30 patients with pneumonic TB, 26 had consolidation on chest radiographs. The radiographic findings in four patients without consolidation included one interstitial, one nodular with cavitation, one nodular and interstitial, and one cavitary. In concordance with the radiographic findings, these patients had severe lung injury (arterial oxygen tension/inspiratory oxygen fraction $\leqslant 200$ and lung injury scores $>2.5$ ). Over $60 \%$ of the patients $(n=25)$ had ARDS. The mean airway pressure was normal. Hypoxaemic respiratory failure was the indication for mechanical ventilation in $11(100 \%)$ patients with miliary TB and in 28 (93\%) patients with tuberculous pneumonia. Only two patients with tuberculous pneumonia were intubated because of hypercapnia.

Among the 41 isolates, seven were drug-resistant. The presence of drug-resistance patterns ranged from single-drug resistance to four-drug resistance, with resistance to single drug being the most common. Four in seven were multidrug resistant. 
Table 1.-Demographic characteristics and disease presentations of 41 intensive care unit patients with severe active pulmonary tuberculosis necessitating mechanical ventilation

\begin{tabular}{|c|c|c|c|c|}
\hline & All & Survivors & Death & p-value \\
\hline Subjects $\mathrm{n}$ & 41 & 14 & 27 & \\
\hline Age yrs & $62.0 \pm 21.8$ & $65.9 \pm 20.4$ & $60.0 \pm 22.6$ & 0.416 \\
\hline Subjects $>65$ yrs of age & $22(53.7)$ & $9(64.3)$ & $13(48.1)$ & 0.326 \\
\hline $\mathrm{M} / \mathrm{F}$ & $34 / 7$ & $13 / 1$ & $21 / 6$ & 0.389 \\
\hline Comorbidities & $20(48.8)$ & $5(35.7)$ & $15(55.6)$ & 0.228 \\
\hline Malignancy & $8(19.5)$ & $3(21.4)$ & $5(18.5)$ & 0.824 \\
\hline $\mathrm{DM}$ & $9(22.0)$ & $3(21.4)$ & $6(22.2)$ & 0.954 \\
\hline Alcoholism & $1(2.4)$ & $1(7.1)$ & 0 & 0.341 \\
\hline Liver cirrhosis & $1(2.4)$ & 0 & $1(3.7)$ & 1.000 \\
\hline ESRD & $1(2.4)$ & 0 & $1(3.7)$ & 1.000 \\
\hline Immunosuppressive therapy & $6(14.6)$ & 0 & $6(22.2)$ & 0.079 \\
\hline Coexisting pulmonary disease & $11(26.8)$ & $4(28.6)$ & $7(25.9)$ & 0.856 \\
\hline History of pulmonary tuberculosis infection & $19(46.3)$ & $6(42.9)$ & $13(48.1)$ & 0.747 \\
\hline \multicolumn{5}{|l|}{ Symptoms } \\
\hline Fever & $30(73.2)$ & $10(71.4)$ & $20(74.1)$ & 0.856 \\
\hline Cough & $28(68.3)$ & $10(71.4)$ & $18(66.7)$ & 0.756 \\
\hline Dyspnoea & $34(82.9)$ & $12(85.7)$ & $22(81.5)$ & 1.000 \\
\hline Haemoptysis & $9(22.0)$ & $3(21.4)$ & $6(22.2)$ & 0.954 \\
\hline Body weight loss & $13(31.7)$ & $6(42.9)$ & $7(25.9)$ & 0.269 \\
\hline Duration of symptom & $9.0 \pm 11.6$ & $8.8 \pm 10.3$ & $9.2 \pm 12.4$ & 0.926 \\
\hline Symptoms $\geqslant 30$ days before admission & $4(9.8)$ & $2(14.3)$ & $2(7.4)$ & 0.596 \\
\hline Extrapulmonary tuberculosis & $10(24.4)$ & $2(14.3)$ & $8(29.6)$ & 0.278 \\
\hline Disseminated tuberculosis & $1(2.4)$ & 0 & $1(3.7)$ & 1.000 \\
\hline \multicolumn{5}{|l|}{ Pattern of pulmonary tuberculosis } \\
\hline Miliary & $11(26.8)$ & $4(28.6)$ & $7(25.9)$ & 0.856 \\
\hline Pneumonia & $30(73.2)$ & $10(71.4)$ & $20(74.1)$ & \\
\hline ARDS & $25(61.0)$ & $5(35.7)$ & $20(61.0)$ & 0.017 \\
\hline APACHE II score & $16.8 \pm 7.5$ & $15.4 \pm 6.1$ & $17.4 \pm 8.2$ & 0.422 \\
\hline Multiple organ failure & $20(48.8)$ & $2(14.3)$ & $18(66.7)$ & 0.001 \\
\hline
\end{tabular}

Data are presented as mean \pm SD or $\mathrm{n}(\%)$ unless otherwise stated. M: male; F: female; DM: diabetes mellitus; ESRD: end-stage renal disease; ARDS: acute respiratory distress syndrome; APACHE: Acute Physiology and Chronic Health Evaluation.

\section{Investigations and outcomes}

Table 4 is the list of in-hospital investigation results. Most patients were diagnosed and treated as non-TB in the beginning. Bacterial pneumonia was the most common diagnosis. Patients with underlying illness $(\mathrm{p}=0.017)$, with symptoms presenting for $<30$ days $(\mathrm{p}=0.008)$ or with pneumonic TB $(\mathrm{p}=0.024)$ were more likely to be misdiagnosed as nontuberculous pulmonary diseases. Misdiagnosis was also more likely to happen among patients with consolidations $(60 \%)$ than among patients with cavitary lesions $(40 \%)$.

Of the 41 patients, $35(85 \%)$ were treated with anti-TB therapy and six $(15 \%)$ were not. All of these six patients had smear-negative tuberculous pneumonia, which had consolidation in more than three quadrants on chest radiographs as its

Table 2.-Radiological features of 41 intensive care unit patients with severe active pulmonary tuberculosis necessitating mechanical ventilation

\begin{tabular}{lccrr}
\hline & All & Survivors & Death & p-value \\
\hline $\begin{array}{l}\text { Subjects n } \\
\text { Pattern of chest radiographs }\end{array}$ & 41 & 14 & 27 & \\
$\quad$ & & & & \\
$\quad$ Nodule & $16(39.0)$ & $7(50.0)$ & $9(33.3)$ & 0.300 \\
$\quad$ Consolidation & $34(82.9)$ & $8(57.1)$ & $26(96.3)$ & 0.004 \\
$\quad$ Interstitial & $10(24.4)$ & $5(35.7)$ & $5(18.5)$ & 0.224 \\
$\quad$ Cavity & $15(36.6)$ & $5(35.7)$ & $10(37.0)$ & 0.934 \\
Extent of lung lesions & & & & \\
$\quad \leq 2$ quadrants & $9(22.0)$ & $5(35.7)$ & $4(14.8)$ & 0.125 \\
$\quad 3$ quadrants & $32(78.0)$ & $9(64.3)$ & $23(85.2)$ & \\
\hline
\end{tabular}

Data are presented as n (\%) unless otherwise stated. major radiographic finding. All had acute onset of symptoms with an average of $6.5 \pm 7.9$ days before admission. The clinical courses were fulminant, with ARDS in four and MOF in five patients.

The mean treatment delay in 35 patients was 15 days. Eight patients received treatment $>1$ month after initial presentation. Patients diagnosed as having nontuberculous pulmonary disease $(\mathrm{p}=0.001)$, with pneumonic TB $(\mathrm{p}=0.04)$ or with consolidation on chest radiographs $(\mathrm{p}=0.036)$ were more likely to have treatment delays of $>1$ month. Thirteen of the 25 patients with ARDS received $\geqslant 2 \mathrm{mg} \cdot \mathrm{kg}^{-1} \cdot \mathrm{day}^{-1}$ methylprednisolone on the seventh day after the onset of ARDS. This was for treatment of the fibroproliferative stage.

Bronchoscopies were conducted on $45 \% \quad(n=18)$ of the

Table 3.-Laboratory parameters of 41 intensive care unit patients with severe active pulmonary tuberculosis necessitating mechanical ventilation

\begin{tabular}{lcccc}
\hline & All & Survivors & Death & p-value \\
\hline Subjects n & 41 & 14 & 27 & \\
WBC & & & & \\
$\quad 4000-12000 \mathrm{~mm}^{-3}$ & $25(61.0)$ & $7(50.0)$ & $18(66.7)$ & 0.300 \\
$\quad<4000$ or $>12000 \mathrm{~mm}^{-3}$ & $16(39.0)$ & $7(50.0)$ & $9(33.3)$ & \\
Positive sputum AFB & $16(39.0)$ & $7(50.0)$ & $9(33.3)$ & 0.300 \\
MDR & $4(9.8)$ & $2(14.3)$ & $2(7.4)$ & 0.759 \\
Initial $P \mathrm{a}, \mathrm{O}_{2} / F \mathrm{I}, \mathrm{O}_{2} \leqslant 200$ & $28(68.3)$ & $8(57.1)$ & $20(74.1)$ & 0.269 \\
Lung injury score $>2.5$ & $17(41.5)$ & $3(21.4)$ & $14(51.9)$ & 0.130 \\
\hline
\end{tabular}

Data are presented as n (\%) unless otherwise stated. WBC: white blood cell; AFB: acid-fast stain; MDR: multidrug resistant; $P \mathrm{a}, \mathrm{O}_{2}$ : arterial oxygen tension; $\mathrm{FI}, \mathrm{O}_{2}$ : inspiratory oxygen fraction. 
Table 4. - Investigations during admission and outcomes of 41 intensive care unit (ICU) patients with severe active pulmonary tuberculosis necessitating mechanical ventilation

\begin{tabular}{|c|c|c|c|c|}
\hline & All & Survivors & Death & $\mathrm{p}$-value \\
\hline Subjects $n$ & 41 & 14 & 27 & \\
\hline \multicolumn{5}{|l|}{ Tentative diagnosis } \\
\hline Pulmonary tuberculosis & $18(43.9)$ & $10(71.4)$ & $8(29.6)$ & 0.011 \\
\hline Nontuberculosis pulmonary disease & $23(56.1)$ & $4(28.6)$ & $19(70.4)$ & \\
\hline Treated before ICU & $12(29.3)$ & $6(42.9)$ & $6(22.2)$ & 0.168 \\
\hline Time to antituberculosis drugs after admission & $14.9 \pm 21.0$ & $8.0 \pm 10.4$ & $19.0 \pm 24.6$ & 0.076 \\
\hline Treatment delay $\geqslant 30$ days & $14(34.1)$ & $1(7.1)$ & $13(48.1)$ & 0.009 \\
\hline Steroid for ARDS & $13(31.7)$ & $4(28.6)$ & $9(33.3)$ & 0.756 \\
\hline Biopsy & $13(31.7)$ & $4(28.6)$ & 9 (33.3) & 0.756 \\
\hline Time to biopsy after admission & $15.3 \pm 14.2$ & $10.5 \pm 8.5$ & $17.8 \pm 16.3$ & 0.431 \\
\hline Bronchoscopy & $18(45.0)$ & $7(50.0)$ & $11(42.3)$ & 0.641 \\
\hline Duration on mechanical ventilation & $29.2 \pm 31.4$ & $29.4 \pm 33.7$ & $29.1 \pm 30.7$ & 0.979 \\
\hline Duration of staying at ICU & $26.9 \pm 24.2$ & $29.6 \pm 24.5$ & $25.4 \pm 24.3$ & 0.601 \\
\hline Bacteraemia & $6(14.6)$ & $2(13.3)$ & $4(14.8)$ & 1.000 \\
\hline Cause of mortality & $27(65.9)$ & & & \\
\hline Hypoxaemia & $10(24.4)$ & & $10(37)$ & \\
\hline Shock & $17(41.5)$ & & $17(67)$ & \\
\hline
\end{tabular}

Data are presented as mean \pm SD or $\mathrm{n}(\%)$ unless stated otherwise. ARDS: acute respiratory distress syndrome.

patients, either because of uncertain diagnosis or suspicions of pulmonary TB with negative smears. Among the 17 specimens obtained from bronchial washing or bronchoalveolar lavage for culture of $M$. tuberculosis, only six (35.3\%) were positive. Biopsies were conducted in 13 patients because of diffuse pulmonary infiltrates and poor responses to antibiotics. These included nine open lung biopsies, three transbronchial lung biopsies and one neck lymph node biopsy. The mean interval between biopsy and admission was 15 days. The pathological findings included eight granulomas, four caseous necrosis, two fibrosis, one giant cell and one inflammation. The acid-fast stains on tissue samples were positive in six. Anti-TB drugs were used on 12 patients after obtaining histopathological proof, except in one patient with pulmonary fibrosis, in whom the lung specimen obtained was from a wedge resection of the middle lobe and showed only fibrosis in the pathology.

Outcomes are listed in table 4 and shown in figures 1 and 2. The in-hospital mortality rate was $65.9 \%$, with 27 of the 41 patients dying in the hospital, while 14 were discharged alive. Twenty-five died during ICU admission and two died after being transferred to the ward. The mean time to death was $40.7 \pm 35.4$ days, with $50 \%$ of the patients expiring within the

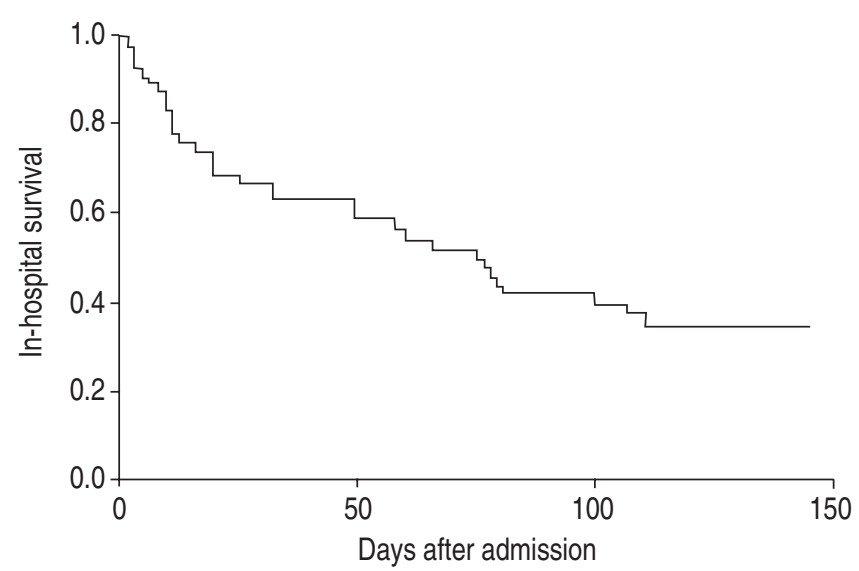

Fig. 1. - In-hospital survival curves by the Kaplan-Meier method and the log-rank test. first 26 days and $75 \%$ of the patients expiring within the first 75 days (fig. 1).

Three of the 14 survival patients failed to follow-up after discharge, the other 11 were followed-up to 180 days. After discharge, three of the 11 patients died and eight survived. The 180 -day mortality of all the 41 patients was $79 \%$. All of the mortality occurred within 150 days.

Of the 35 patients who took anti-TB therapy, 24 died while eight survived and three were lost to follow-up. Twenty-two of 24 died during hospitalisation and two died after discharge. All of the six patients not treated with anti-TB drugs died, five in-hospital and one after discharge. The mean time to death was significantly shorter in nontreated patients (17.6 \pm 11.6 days) than in patients who received anti-TB treatment $(46.0 \pm 37.0$ days; $\mathrm{p}=0.006$ ).

The mean length of ICU stay was 26.9 days. The mean duration of mechanical ventilation was 29.2 days. Nine of 14 patients were successfully weaned from ventilators before discharge. The other five patients were still dependent on ventilators on day 180 .

\section{Factors predicting in-hospital mortality}

Five factors were found to be associated with in-hospital mortality in the univariate analysis (tables 1-4). They were tentative diagnosis as nontuberculous pulmonary disease, ARDS, consolidation on chest radiographs, MOF and treatment delays $\geqslant 30$ days. The Kaplan-Meier survival curves (fig. 2) disclosed a trend of higher mortality rate among patients with ARDS ( $\mathrm{p}=0.0196)$, consolidation $(\mathrm{p}=0.0139)$ and MOF ( $\mathrm{p}=0.0099)$.

The Chi-squared test revealed high correlations between the tentative diagnosis of nontuberculous pulmonary disease and MOF $(\mathrm{p}=0.0003)$ and treatment delay $(\mathrm{p}=0.0006)$, and between MOF and treatment delays $(\mathrm{p}<0.0001)$. There was moderate correlation between consolidation and treatment delays ( $\mathrm{p}=0.0364)$. However, the correlations between ARDS, consolidation and MOF were not statistically significant. The ARDS, consolidation and MOF correlations were introduced into the stepwise Cox regression model, which showed that consolidation (HR 7.731, 95\% CI 1.036-57.68, $\mathrm{p}=0.046$ ) and MOF (HR 2.651, 95\% CI 1.163-6.04, $\mathrm{p}=0.020$ ) were factors affecting the in-hospital mortality rate (table 5). 

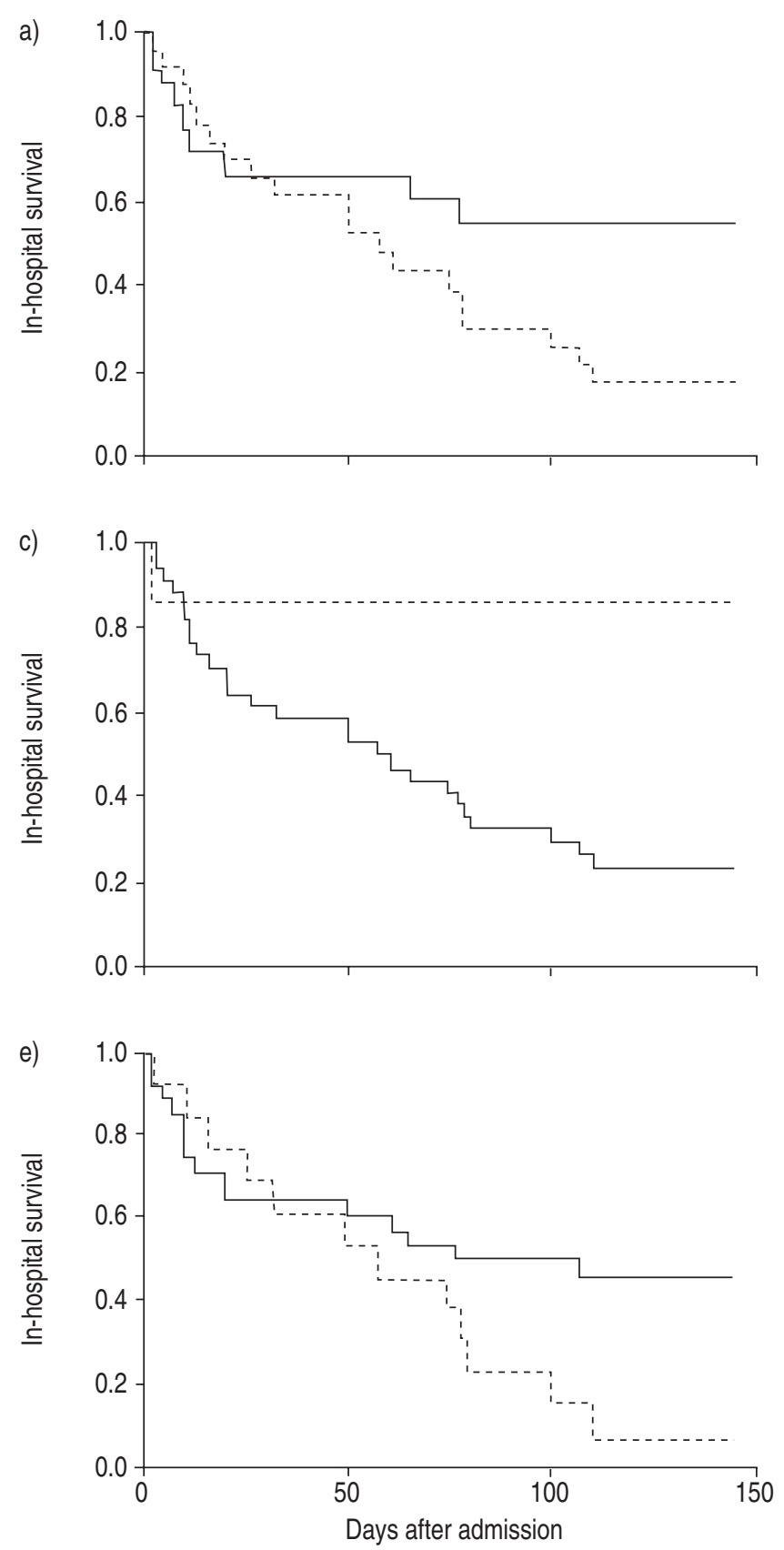

\section{Discussion}

This study sought to identify factors predicting in-hospital mortality in ICU patients who had active pulmonary TB as the primary cause of acute respiratory failure and required mechanical ventilation. Forty-one cases formed the basis

Table 5.-Cox-proportional hazard model of factors contributing to intrahospital mortality

\begin{tabular}{lccc}
\hline & \multicolumn{3}{c}{ Multivariable logistic regression model } \\
\cline { 2 - 4 } Variable & HR & $95 \%$ CI & p-value \\
\hline Consolidation & 7.731 & $1.036-57.680$ & 0.046 \\
Multiple organ failure & 2.651 & $1.163-6.040$ & 0.020 \\
\hline
\end{tabular}

HR: hazard ratio; CI: confidence interval. b)

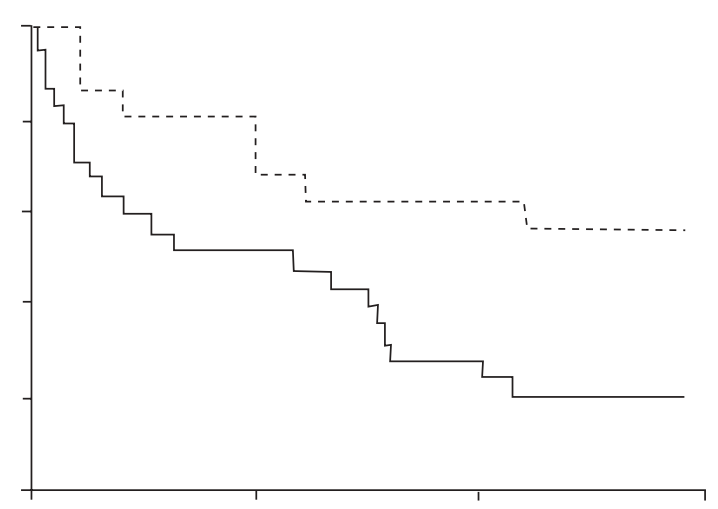

d)

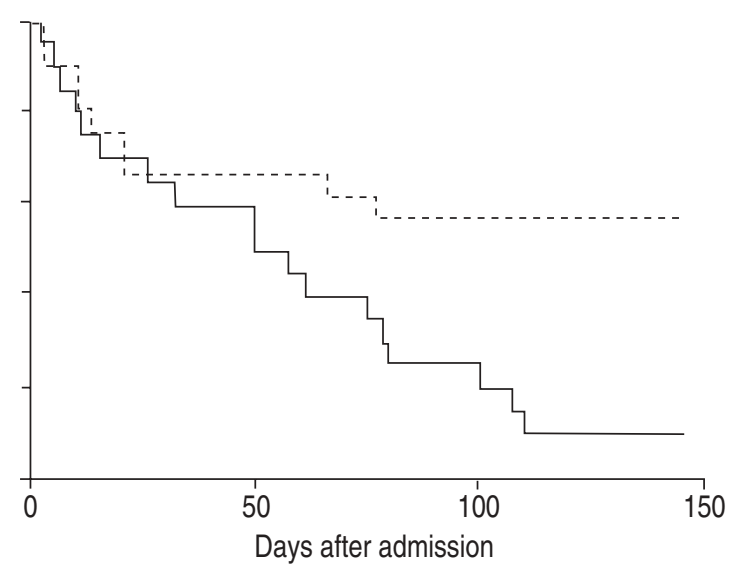

Fig. 2. - Kaplan-Meier survival curves according to the absence (- - -) or presence (-) of predictors of in-hospital mortality. a) Tentative diagnosis of pulmonary tuberculosis at arrival, $p=0.052$. b) Acute respiratory distress syndrome, $\mathrm{p}=0.0196$. c) Consolidation on radiographs, $\mathrm{p}=0.0139$. d) Multiple organ failure, $\mathrm{p}=0.0099$. e) Treatment delays of $\geqslant 30$ days, $p=0.06$.

of the study. The ICU mortality rate was $61 \%$, with an in-hospital mortality rate of $65.9 \%$ and a 180 -day mortality rate of $79 \%$. After adjusting for potential confounding effects, two factors contributing to in-hospital mortality were identified, MOF and consolidation on chest radiographs.

Even though the duration of mechanical ventilation and ICU stay in the study was twice as long as other studies, the in-hospital and ICU mortality rate was similar to, or even higher than, previous reports $[4-6,8,10]$. In one study, the 180-day mortality rate was $\sim 60 \%$, with nearly all deaths occurring within the first 2 months of hospital admission [10]. In contrast, the 180-day mortality rate in the study was as high as $79 \%$, with one-half of the deaths occurring in the first 2 months and the remaining deaths occurring in the following 3 months of hospital stay. Both 30-day and 180-day mortality rates were obviously far higher than the mortality rate among patients with nontuberculous pneumonia requiring mechanical ventilation $[4,11,12]$. 
In this study, pneumonic TB was twice as frequent as miliary $\mathrm{TB}$, which was in contrast to what has been reported in the previous medical literature $[4,5,22]$. In the 30 patients with pneumonic TB, 26 had consolidation in their chest radiographs. The radiographic findings in the four patients without consolidation included one interstitial, one nodular with cavitation, one nodular with interstitial and one cavitary. Of these 30 patients, $28(93 \%)$ were intubated because of hypoxaemic respiratory failure, while the other two were due to hypercapnia. This finding suggests that the primary cause of respiratory failure in patients with pneumonic TB in this study was severe damage to the lung parenchyma, not obstructed airways.

Consolidation was a strong independent factor contributing to in-hospital death. In contrast, the percentage of patients with cavitary, interstitial or nodular patterns was the same among the fatalities and the survivors. These findings were similar to other studies discussing predictors of in-hospital death or short-term prognosis for patients with pulmonary TB $[8,15,16]$. It is possible that clinicians were prone to favour the diagnosis of nontuberculous pulmonary disease if radiographs disclosed multiple consolidations, particularly for patients with immunodeficiency, resulting in a delay in antituberculous treatment [8, 13-15, 17-19]. Another possible reason was that consolidation suggests an inadequate immune response to a heavy burden of TB bacilli [16], and death was a consequence of poor immunity.

In the present study, patients with MOF had higher mortality rates than those without. The finding was similar to other studies examining ICU patients with active pulmonary TB and acute respiratory failure [4, 8]. The higher number of organ failures reflects the propensity of uncontrolled, untreated infection to cause multiple organ dysfunction. However, the APACHE II scores of those who expired were similar to those in the survivors. In this study, the mean APACHE II score was $16.8 \pm 7.5$, which should have reflected a mortality rate of $20-30 \%$ in patients with respiratory failure from infection [26]. However, the exact mortality rate was $65.9 \%$. This finding suggested that the APACHE II score consistently underestimated mortality of patients with pulmonary TB $[8,27]$. Meanwhile, organ failure scores were also proven to be superior to a single APACHE II assessment, or daily APACHE II scores, in predicting the outcome among ICU patients [24, 25]. Therefore, for patients with pulmonary $\mathrm{TB}$ and acute respiratory failure, computerised daily organ failure scores should be applied instead of a single APACHE II assessment.

The possible causes for misdiagnosing pulmonary TB as a nonpulmonary disease include atypical chest radiographs and the short duration of symptoms. The mean duration of symptoms in this study was only $9.0 \pm 11.6$ days, which was far shorter than was normally reported and may have contributed to the misdiagnoses. The lack of significant respiratory symptoms and insidious constitutional symptoms may also cause a doctor to overlook the duration of symptoms [4, 13]. Pulmonary TB is more likely to be suspected in patients with cavitary lesions than in those who display other radiological presentations [8, 13-15, 18-22]. The present results support this finding. However, consolidation is a far more common presentation than cavitation, which contributed to the misdiagnosis. For example, in six patients not treated with anti-TB medications during hospitalisation, the duration of symptoms was short and all had pneumonic TB, with consolidation in more than three quadrants on their radiographs as the main radiographic findings. This made an accurate diagnosis more difficult, which in turn contributed to delays in treatment.

Timely treatment has long been considered a crucial factor in a patient's ability to survive TB [2, 4, 8, 13-15]. In this study, the trend of a higher mortality rate in patients with treatment delays is clear though it did not reach statistical significance. The small patient number and interdependence with other factors contributed to the statistical insignificance. Factors contributing to treatment delays included the presence of consolidation and the presence of pneumonic TB, both of which lead to misdiagnosis. The misdiagnosis is also highly related to treatment delays. Therefore, the authors urge the promotion of early diagnosis to be crucial for decreasing the delays in treatment.

MDR was identified as a dominant factor affecting the survival of patients with TB in a New York study [2], but not in the present study. The median time to death was 6.3 months for the New York City patients, compared with only 40.7 \pm 35.4 days in this study. Due to the shorter median time to death, the present authors' study on in-hospital mortality has revealed a different spectrum of clinical issues causing early death from TB without the influence of MDR [15].

Though HIV infection was not an exclusion criterion in this study, none of the 41 TBMV patients were HIV-positive. However, the mortality rate of the current study was similar to other studies, including those with HIV patients [4-7]. It is worth noting that the early mortality rate from TB in HIVpositive patients was not different from that of HIV-negative patients [28]. The mean time to expiration in the current patients was only 56 days, which is not enough time for HIV to become a significant causal factor of mortality [29].

Other studies of in-hospital TB mortality took into account factors such as age, sex, haemoglobin level, weight, alcoholism, lymphopaenia and end-stage renal disease $[4,10,13,16,30]$. However, these studies fail to distinguish between pulmonary and extrapulmonary $\mathrm{TB}$, and most are either descriptive or rely only on univariate tests. In contrast, the present study focused on patients with pulmonary TB as the primary cause of respiratory failure. All of the factors were analysed using multivariable analysis and Kaplan-Meier methods, so it is not surprising that these factors had little or no impact on mortality in the study.

In conclusion, the mortality rate of patients with active pulmonary tuberculosis requiring mechanical ventilation is high, especially in patients with consolidation on chest radiographs and with multiple organ failure. Therefore, the authors recommend that intensive care unit patients with cough, dyspnoea and fever without evidence of a documented infective microorganism, promptly receive an aggressive search of aetiology to exclude tuberculosis infection, especially for patients with the presence of consolidation and multiple organ failure.

\begin{abstract}
Acknowledgements. This study would not have been possible without the substantial and invaluable contribution of time and talent from P-C. Chen and $\mathrm{W}-\mathrm{Y}$. Shau who provided help with the statistical analysis.
\end{abstract}

\section{References}

1. Raviglione MC, Snider DE, Kochi A. Global epidemiology of tuberculosis: morbidity and mortality of a worldwide epidemic. JAMA 1995; 273: 220-226.

2. Pablos-Méndez A, Sterling TR, Frieden TR. The relationship between delayed or incomplete treatment and all-cause mortality in patients with tuberculosis. JAMA 1996; 276: 1223-1228.

3. Dolin PJ, Rariglione MC, Kochi A. Global tuberculosis incidence and mortality during 1990-2000. Bull World Health Organ 1994; 72: 213-220.

4. Penner C, Roberts D, Kunimoto D, Manfreda J, Long R. Tuberculosis as a primary cause of respiratory failure requiring mechanical ventilation. Am J Respir Crit Care Med 1995; 151: 867-872.

5. Levy H, Kallenbach JM, Feldman C, Thorburn JR, 
Abramowitz JA. Acute respiratory failure in active tuberculosis. Crit Care Med 1987; 15: 221-225.

6. Agarwal MK, Muthuswamy PP, Banner AS, Shah RS, Addington WW. Respiratory failure in pulmonary tuberculosis. Chest 1977; 72: 605-609.

7. Frame RN, Johnson MC, Eichenhorn MS, Bower GC, Popovich J. Active tuberculosis in the medical intensive care unit: a 15-year retrospective analysis. Crit Care Med 1987; 15: 1012-1014.

8. Zahar JR, Azoulay E, Klement E, et al. Delayed treatment contributes to mortality in ICU patients with severe active pulmonary tuberculosis and acute respiratory failure. Intensive Care Med 2001; 27: 513-520.

9. Vyskocil JJ, Marik P, Greville HW. Survival with tuberculosis pneumonia necessitating mechanical ventilation. Clin Pulm Med 1995; 2: 152-156.

10. Rao VK, Iademarco EP, Fraser VJ, Kollef MH. The impact of co-morbidity on mortality following in-hospital diagnosis of tuberculosis. Chest 1998; 114: 1244-1252.

11. Confalonieri M, Potena A, Carbone G, Porta RD, Tolley EA, Meduri GU. Acute respiratory failure in patients with severe community-acquired pneumonia. A prospective randomized evaluation of noninvasive ventilation. Am J Respir Crit Care Med 1999; 160: 1585-1591.

12. Jolliet P, Abajo B, Pasquina P, Chevrolet JC. Non-invasive pressure support ventilation in severe community-acquired pneumonia. Intensive Care Med 2001; 27: 812-821.

13. Mathur P, Sacks L, Auten G, Sall R, Levy C, Gordin F. Delayed diagnosis of pulmonary tuberculosis in city hospitals. Arch Intern Med 1994; 154: 306-310.

14. Katz I, Rosenthal T, Michaeli D. Undiagnosed tuberculosis in hospitalized patients. Chest 1985; 87: 770-774.

15. Sacks LV, Pendle S. Factors related to in-hospital deaths in patients with tuberculosis. Arch Intern Med 1998; 158: 19161922.

16. Barnes PF, Leedom JM, Chan LS, et al. Predictors of shortterm prognosis in patients with pulmonary tuberculosis J Infect Dis 1988; 158: 366-371.

17. Bobrowitz ID. Active tuberculosis until autopsy. Am J Med 1982; 72: 650-658.

18. Rao VK, Iademarco EP, Fraser VJ, Kollef MH. Delays in the suspicion and treatment of tuberculosis among hospitalized patients. Ann Intern Med 1999; 130: 404 411.
19. Dyer RA, Potgieter PD. The adult respiratory distress syndrome and bronchogenic pulmonary tuberculosis. Thorax 1984; 39: 383-387.

20. Miller WT, MacGregor RR. Tuberculosis: frequency of unusual radiographic findings. Am J Roentgenol 1978; 130: 867-875.

21. Gordin FM, Slutkin G, Schecter G, Goodman PC, Hopewell PC. Presumptive diagnosis of and treatment of pulmonary tuberculosis based on radiographic findings. Am Rev Respir Dis 1989; 139: 1090-1093.

22. Choi D, Lee KS, Suh GY, et al. Pulmonary tuberculosis presenting as acute respiratory failure: radiologic findings. J Comput Assist Tomogr 1999; 23: 107-113.

23. Bernard GR, Artigas A, Brigham KL, et al. The AmericanEuropean Consensus Conference on ARDS: definitions, mechanisms, relevant outcomes, and clinical trial coordination. Am J Respir Crit Care Med 1994; 149: 818-824.

24. Knaus WA, Draper EA, Wagner DP, Zimmerman JE. Prognosis in acute organ-system failure. Ann Surg 1985; 202: 685-693.

25. Chang RWS, Jacobs S, Lee B. Predicting outcome among intensive care unit patients using computerized trend analysis of daily Apache II scores corrected for organ system failure. Intensive Care Med 1988; 14: 558-566.

26. Knaus WA, Draper EA, Wagner DP, Zimmerman JE. APACHE II: a severity of disease classification system. Crit Care Med 1985; 13: 818-829.

27. Root A, Smith C, Feldman C. APACHE II severity of illness score in patients with severe active pulmonary tuberculosis. $S$ Afr J Crit Care 1990; 6: 13-14.

28. Nunn P, Brindle R, Carpenter L, et al. Cohort study of HIV infection in patients with tuberculosis in Nairobi, Kenya: analysis of early (6 month) mortality. Am Rev Respir Dis 1992; 146: 849-854.

29. Stoneburner R, Laroche E, Prevots R, et al. Survival in a cohort of human immunodeficiency virus-infected tuberculosis patients in New York City: implications for the expansion of the AIDS case definition. Arch Intern Med 1992; 152: 2033-2037.

30. Humphries MJ, Byfield SP, Darbyshire JH, et al. Deaths occurring in newly notified patients with pulmonary tuberculosis in England and Wales. Br J Dis Chest 1984; 78: $149-158$ 